Precision timing in ocean sensor systems

This article has been downloaded from IOPscience. Please scroll down to see the full text article.

2012 Meas. Sci. Technol. 23025801

(http://iopscience.iop.org/0957-0233/23/2/025801)

View the table of contents for this issue, or go to the journal homepage for more

Download details:

IP Address: 147.83.82.7

The article was downloaded on 17/12/2011 at 10:59

Please note that terms and conditions apply. 


\title{
Precision timing in ocean sensor systems
}

\author{
Joaquín del Río ${ }^{1}$, Daniel Toma ${ }^{1}$, Shahram Shariat-Panahi ${ }^{1}$, \\ Antoni Mànuel ${ }^{1}$ and Helena Geirinhas Ramos ${ }^{2}$ \\ ${ }^{1}$ SARTI Research Group, Electronics Department, Universitat Politècnica de Catalunya, UPC, \\ Barcelona, Spain \\ ${ }^{2}$ Instituto de Telecomunicaçoes, DEEC, Instituto Superior Tecnico, UTL, Lisboa, Portugal \\ E-mail: Joaquin.del.rio@upc.edu and hgramos@1x.it.pt
}

Received 14 June 2011, in final form 30 October 2011

Published 16 December 2011

Online at stacks.iop.org/MST/23/025801

\begin{abstract}
This paper discusses the use of the IEEE 1588 standard in ocean observatories. The performance result of prototype implementations of this standard in an Ethernet Marine Sensor Network (MSN) is presented. The performance tests emulate an underwater-cabled observatory with a Master Clock synchronized with GPS, located in an on-shore station, and with underwater instruments requiring high-precision PPS (pulse $\mathrm{s}^{-1}$ ) signals for synchronization purposes. These signals will be provided to the underwater station by an IEEE 1588 GPS Emulator connected to the observatory's Local Area Network (LAN). The experimental setup emulates the underwater-cabled observatory OBSEA where this technology will be installed due to synchronization requirements of marine instruments such as ocean bottom seismometers.
\end{abstract}

Keywords: IEEE 1588, ocean observatories, time synchronization

(Some figures in this article are in colour only in the electronic version)

\section{Introduction}

Ocean observatories are an important technological resource that can provide knowledge to forecast environment changes or to carry out ocean research [1-3]. Sensor data are collected at different world locations and large streams of data must be correlated with other events or data sources to study either long-term tendencies or singular events [4]. Data in a location context must be time-stamped in order to allow this analysis $[5,6]$. For many observations, low time-stamp accuracy is adequate, whereas for other observations, sub-millisecond precision level is needed.

OBSEA is a cabled seafloor observatory $4 \mathrm{~km}$ offshore Vilanova i la Geltrú (Barcelona, Spain) at $20 \mathrm{~m}$ water depth, interconnected to the coast by an energy and communication mixed cable [7]. As an underwater observatory, GPS (Global Positioning System) signals are unavailable due to seawater attenuation at the sea bottom. For this reason, IEEE Std 1588 was the protocol chosen to distribute time to the subsea instruments that make up the sea observatory. IEEE 1588, also known as the 'Precision Time Protocol (PTP), defines a method to synchronize real-time clocks in the nodes of a distributed system that communicates using standardized packet-based networks such as Ethernet [8]. Its usage on CAN bus [9] or wireless networks [10] has been documented in recent reports. PTP is intended for relatively localized systems in measurement environments, its installation is administration free and it has minimal resource requirements in networks and host components. The time signal delivered to the application depends on the quality of the master clock and the application service interface allowing all nodes to synchronize in the submillisecond range. This is the precision required for timestamping some data collected at OBSEA. For instance, in order to locate an earthquake epicenter, or the position of a sound source installed in a boat or in an animal, the triangularization of three measurements performed at the same moment is needed. The resultant accuracy depends on the quantities measured (from seismometers [11] or from hydrophones [12]) and on their time-stamping accuracies. Other wellknown synchronization protocols such as NTP (Network Time Protocol) or SNTP (Single Network Time Protocol) offer clock synchronization with accuracies around milliseconds far away from accuracies offered by PTP.

This paper describes the project and implementation of timing signals delivered to the cabled ocean observatory OBSEA using IEEE 1588 PTP [13, 14]. The accuracy 


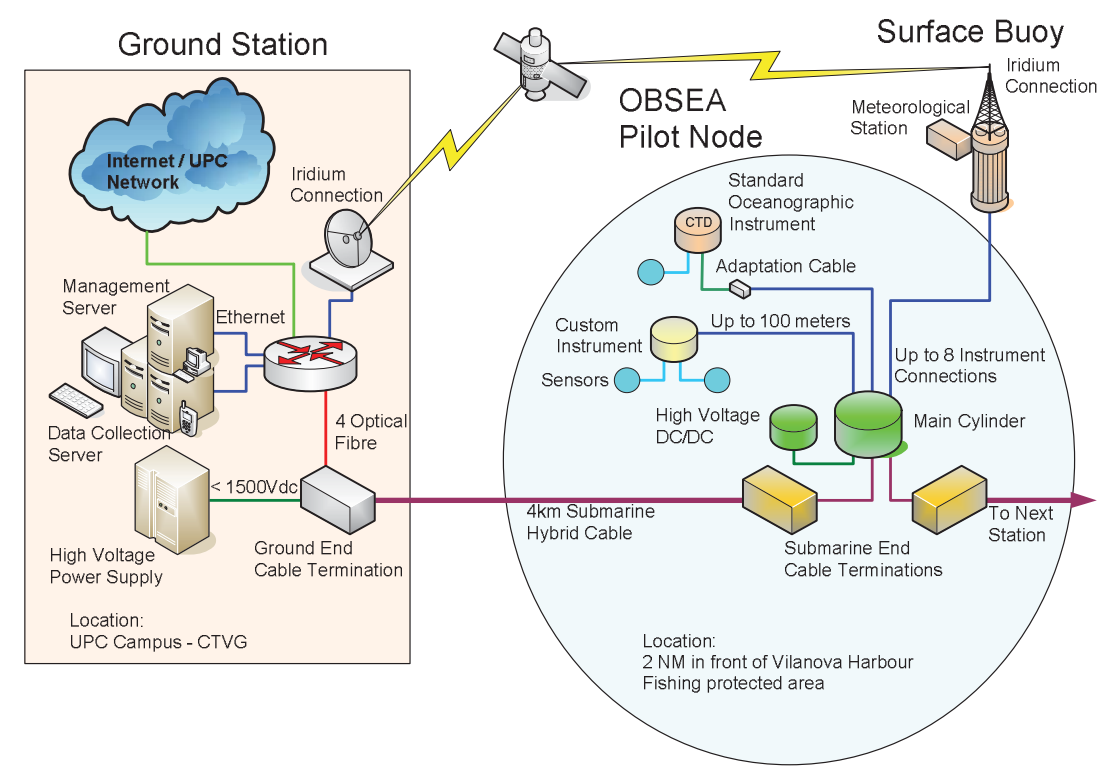

Figure 1. Block diagram of the OBSEA observatory.

achieved with PTP implementation depends greatly on the inclusion of specialized timing hardware at the network interface (PTP-enabled switches). OBSEA was deployed before IEEE 1588v2 was released and as the junction boxes are not equipped with IEEE 1588 Ethernet switches, the evaluation of the time synchronization accuracy achieved within the network done within this paper is thus of paramount importance. The performance evaluation of a testing setup installed in the laboratory simulating the real situation is described in detail and conservative limits for the precision achieved are presented.

The paper is divided into five parts. The following section describes the system architecture. Section 3 gives an overview of the IEEE 1588 standard. The experimental tests are reported in section 4 and the results are presented and analyzed in section 5. Section 6 presents the major conclusions.

\section{System architecture}

\subsection{OBSEA timing requirements}

The OBSEA infrastructure contains two main installations, the ground station and the subsea station, connected with a cable containing the power and data channels as depicted in the functional block diagram presented in figure 1. The subsea station has all the oceanographic instruments and related electronics for power supply, data acquisition, communications and control. All the instruments at the subsea station are transparently accessible through a TCP/IP connection. The ground station includes the management servers that continuously store information and give control access to the scientific community. A primary clock is located at the ground station and is synchronized to the coordinated Universal clock (UTC) by means of a global positioning system time signal (GPS).

Several options can apply for the time distribution in a cabled ocean observatory like OBSEA: (1) a timing signal can be broadcast to be received by all nodes; (2) a dedicated timing channel can be added to the power and data buses built in the cable that links the subsea and the ground station; (3) a clock signal across the distributed network of Ethernet. The most advantageous solution is the last one as minimal costs are required as it can operate over the same communication channels that carry data and without adding any equipment to the observatory infrastructure. This was the solution proposed for OBSEA and IEEE 1588 was the protocol chosen to be implemented as it is the most accurate over packet-switched, variable latency data networks such as Ethernet.

\section{Method}

\subsection{IEEE 1588 overview}

IEEE 1588 is a standard that specifies a protocol to synchronize independent clocks running on separate nodes of a distributed relatively localized network. The clocks communicate with each other through a bidirectional multicast communication and four 1588 messages are necessary for the synchronization process: Sync, Follow_up, Delay_Req and Delay_Resp. The messaging between the master and the slave clocks is depicted in figure 2.

For a slave node to synchronize with a master node, two quantities must be determined: (1) the slave node's clock offset in relation to the master's clock (offset) and (2) the network propagation delay (delay). Considering that the master-toslave delay is equal to the slave-to-master delay and by using the four messages illustrated in figure 2 , these quantities can be calculated from the four measured values $t_{1}, t_{2}, t_{3}$ and $t_{4}$ :

$$
\begin{gathered}
\text { delay }=\frac{\left(t_{2}-t_{1}\right)+\left(t_{4}-t_{3}\right)}{2} \\
\text { offset }=t_{2}-t_{1}-\text { delay. }
\end{gathered}
$$




\section{Master Clock Time}

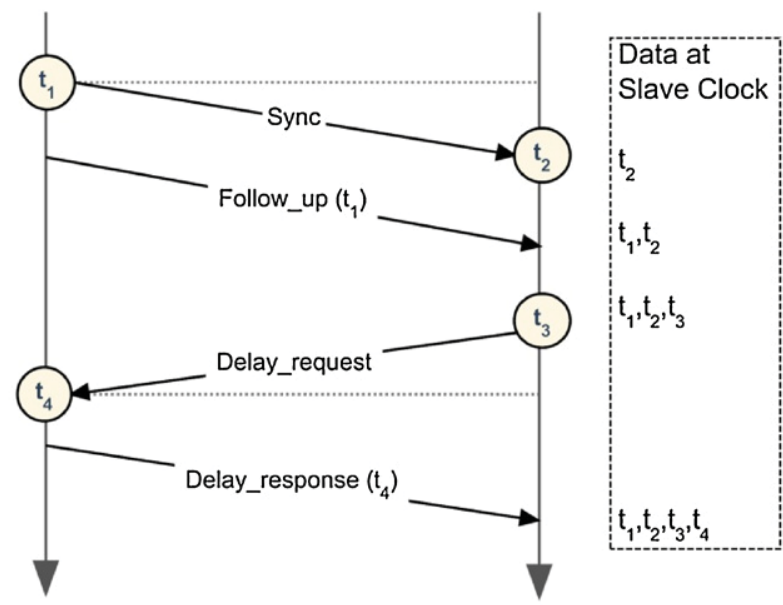

Figure 2. The IEEE 1588 time-synchronization message exchange sequence.

\subsection{Protocol implementation}

The protocol IEEE 1588 can be implemented in two different approaches: software-only or hardware-assist. In softwareonly implementation, the protocol is wholly executed at the application level and the accuracy will depend mainly on software and how the hardware layer executes the firmware source code. As the hardware-assisted implementation delivers more precise time synchronization, it was the solution chosen for OBSEA.

Both microcontrollers from Stellaris (Texas Instruments) and Imsys $\mathrm{AB}$ manufacturers with hardware-assisted support for synchronization using the IEEE 1588 Precision Time Protocol have been used with different protocol implementation. Both Imsys IM3200 and Stellaris microcontrollers can execute IEEE 1588 protocol versions 1 and 2, stack implementation from the Zurich University of Applied Sciences (ZHAW) [14]. In addition, an open source stack of IEEE 1588v1 named PTPd [13] can be executed on Stellaris microcontrollers. The experimental setup and results presented in this paper have been done using the PTPd implementation. Previous results using Imsys IM3200 have been published [15].

The PTPd open source stack has been used to develop an underwater GPS Emulator installed at OBSEA providing timing synchronization to an underwater seismometer. Tests have been carried out for the Imsys microcontroller and ZHAW implementation for the Stellaris microcontroller to compare the time synchronization performance for the different implementation and IEEE 1588 protocol version 2.

\section{Experimental setup}

In order to evaluate the performance of the Precision Time Protocol for network node synchronization, the demonstrative test setup illustrated in figure 3 was installed in the laboratory.
This test setup reproduces the OBSEA network where a primary master clock is located at the on-shore station and will be synchronized to the UTC by means of a GPS and the slave clocks, synchronized within sub-millisecond accuracy, are located at the subsea observatory nodes. Between the master and the slave, there is a maximum of two Ethernet switches. Both switches are commercial off-the-shelf (COTS) equipment without PTP hardware support.

The tests carried out compare two PPS, one generated at the ground station (F1) and another at the subsea node (F2), under different working conditions.

\subsection{Hardware description}

The demonstrative test setup used to evaluate the performance of the IEEE 1588 protocol when non-PTP Ethernet Switches are present at the network is illustrated in figure 3 .

The on-shore station of the test setup includes a personal computer (PC) with a National Instruments PCI board (NIPCI-1588) working as the Master Clock in the system that generates a pulse-per-second (PPS) signal (F1) via a general purpose Programmable Function Interface (PFI) line. The NI-PCI-1588 uses the IEEE 1588 precision time protocol to synchronize clocks and events with other IEEE 1588based instruments, computers or embedded systems [14] on the LAN. The LAN is composed of two non-1588 Ethernet Switches that connect ground and subsea stations with CAT 5 cabling.

At the off-shore underwater station, the GPS emulator is implemented using a LM3S8962 Stellaris Luminary microcontroller with IEEE 1588 protocol capability, able to generate a second PPS signal (F2). This PPS signal will be used further on to provide synchronization to the instruments that are included at the subsea station. The Stellaris microcontroller runs RISC OS at $50 \mathrm{MHz}$ on a 32 bit $\mathrm{ARM}^{\circledR}{ }^{\circledR}$ Corte $^{\mathrm{TM}}{ }^{\mathrm{T}}$-M3 chipset. It possesses the 10/100 Ethernet controller with a fully integrated media access controller (MAC) and network physical interface (PHY). Besides its high configurability capability, it provides highly accurate time stamps for individual packets. Other benefits of the Stellaris-assisted IEEE 1588 PTP system include absolute time base (as in the real world), conventional and inexpensive network components, fault tolerance, self-configuration and hot-plugging capability.

In order to measure the delays between the two PPS signals, F1 and F2, an oscilloscope, TDS-784D from Tektronix, and a computer are used. The PC runs a LabVIEW application from National Instruments that stores the measurements in a data file. The TDS 784D Oscilloscope has an accuracy of \pm 200 ps. The drift between the two PPS signals has been measured using the delay function of the oscilloscope and the F1 PPS signal was used as the reference.

\subsection{Software layer}

The software running in the Stellaris microcontroller which implements the IEEE 1588 standard is based on the open source stacks $l w I P$ and PTPd. Figure 4 shows the block diagram of the implementation used to run the PTP IEEE 


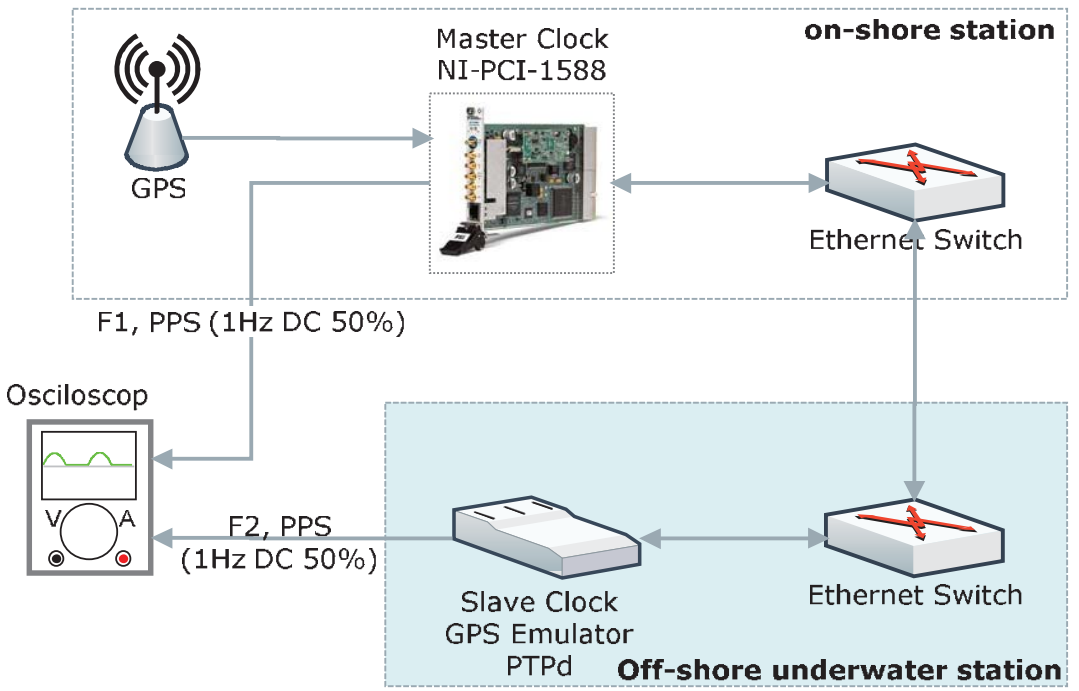

Figure 3. Setup for F1 to F2 delay measurements.

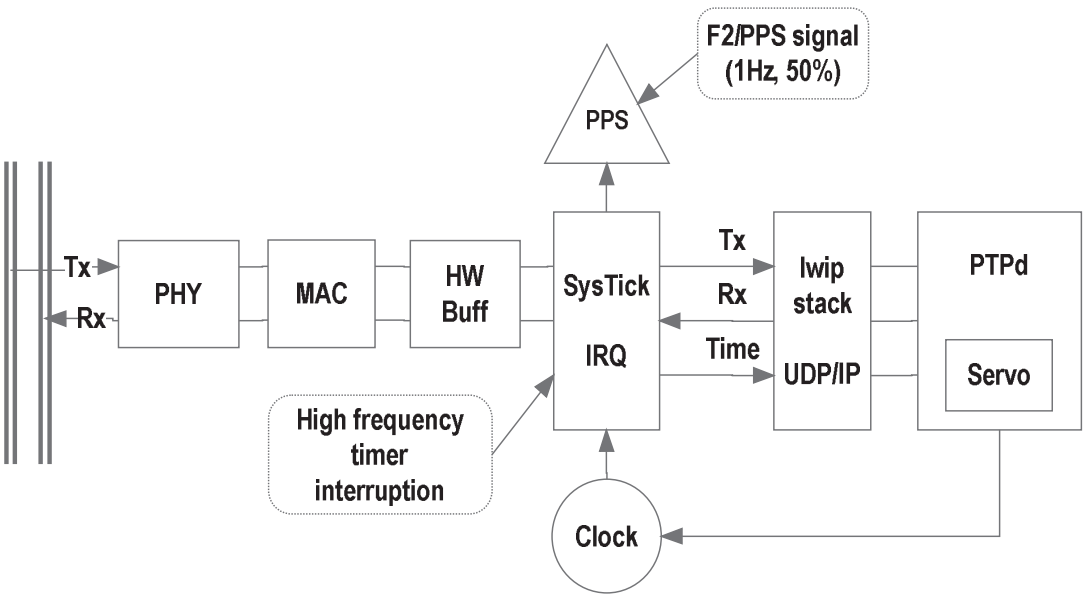

Figure 4. Stellaris system running PTPd. Block diagram of PTP implementation and PPS generation.

1588 protocol and to generate the F2 PPS signal. The PPS signal is generated using a GPIO pin that goes from ' 0 ' to ' 1 ' every $500 \mathrm{~ms}$ (1PPS).

The Master Clock is activated by running the 1588 LabVIEW Application Programming Interface (API) from the NI Sync hardware driver. The PTP synchronization protocol and the F1 PPS signal were generated using the NI-PCI-1588 card. Table 1 flowcharts the LabVIEW application used to configure and generate a PPS signal with a frequency of $1 \mathrm{~Hz}$ and a $50 \%$ duty cycle.

F1 and F2 have the same frequency $(1 \mathrm{~Hz})$ and duty cycle (50\%) parameters. The PTP synchronization engine had to be started in both sources before recording the measurement delays.

Windows software was used to generate the network payload. The software is designed to generate and monitor IP/ICMP/TCP/UDP traffic from clients to servers and to stress test routers, servers and firewalls under extreme network loads.
Table 1. Flowchart of the LabVIEW program to configure and generate F1.

\begin{tabular}{|c|c|}
\hline Step & Description \\
\hline 1 & Create a new NI-SYNC driver session \\
\hline 2 & Read the current 1588 time \\
\hline 3 & $\begin{array}{l}\text { Define when the clock signal will be generated by } \\
\text { adding a delay to the current } 1588 \text { time and } \\
\text { rounding up to the nearest seconds' boundary }\end{array}$ \\
\hline 4 & $\begin{array}{l}\text { Program the frequency of } \mathrm{F} 1 \text { to } 1 \mathrm{~Hz} \text { and the start } \\
\text { time of the clock to be generated }\end{array}$ \\
\hline 5 & Display the current 1588 time \\
\hline 6 & $\begin{array}{l}\text { Clear the clock which stops the F1 PPS clock } \\
\text { generation and release the resource }\end{array}$ \\
\hline 7 & Close the driver session \\
\hline
\end{tabular}

\section{Experimental results}

\subsection{Drift without PTP}

In order to justify the need for a time synchronization protocol, the time delay between two PPS signals (F1 and F2) with a 


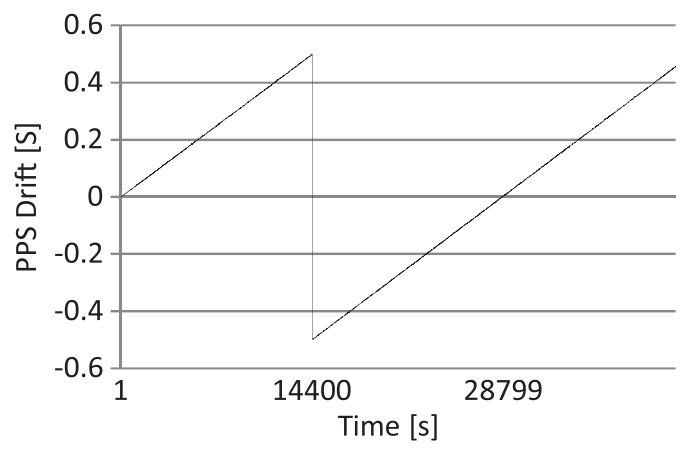

Figure 5. PPS signal delay evolution without PTP IEEE 1588 synchronization.

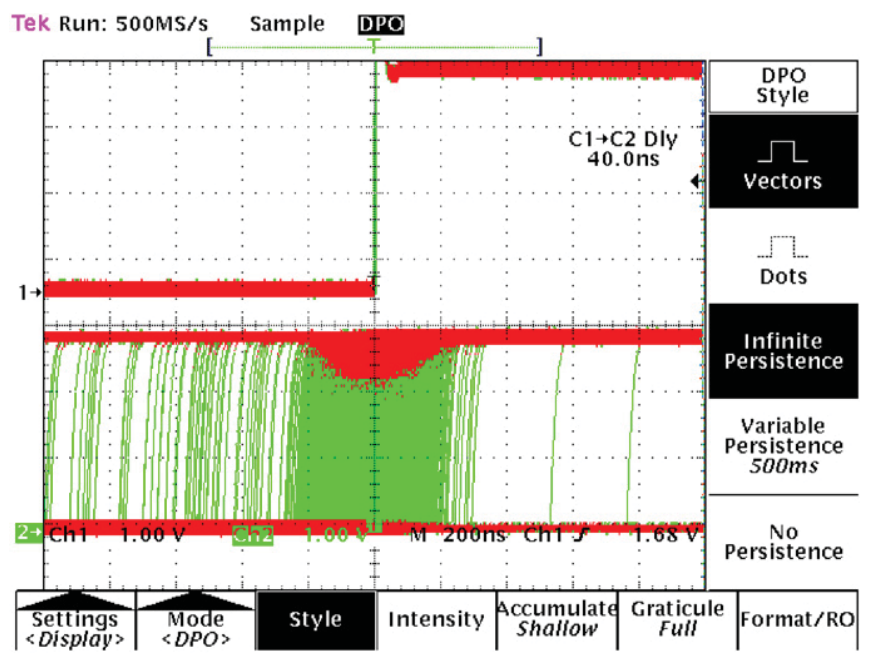

Figure 6. PTP experiment results. Note that the horizontal scale is $200 \mathrm{~ns}$ per division.

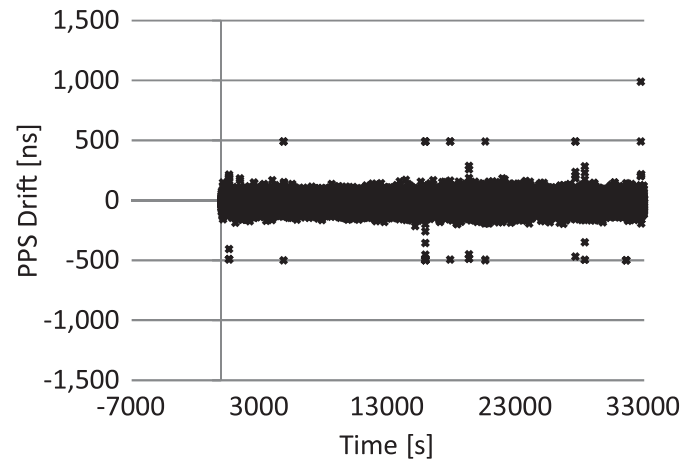

Figure 7. PPS signal delay evolution with PTP IEEE 1588 using COTS Ethernet Switch without traffic.

frequency of $1 \mathrm{~Hz}$ and duty cycle $50 \%$, generated respectively by the Luminary Microcontroller and by the PCI-1588 card, is measured. The results obtained for the clock drift are presented below.

Figure 5 shows how the delay increases as a function of time when the synchronization protocol is not implemented. Each platform generated a $1 \mathrm{~Hz}$ clock signal and due to drifts in their main clocks, the signals are drifting from one other. As depicted, the clock drifts $0.5 \mathrm{~s}$ for every $14400 \mathrm{~s}$ ( $1 \mathrm{~s}$ for every $28800 \mathrm{~s}$ ). This represents a drift of about $34 \mu \mathrm{s} \mathrm{s}^{-1}$.

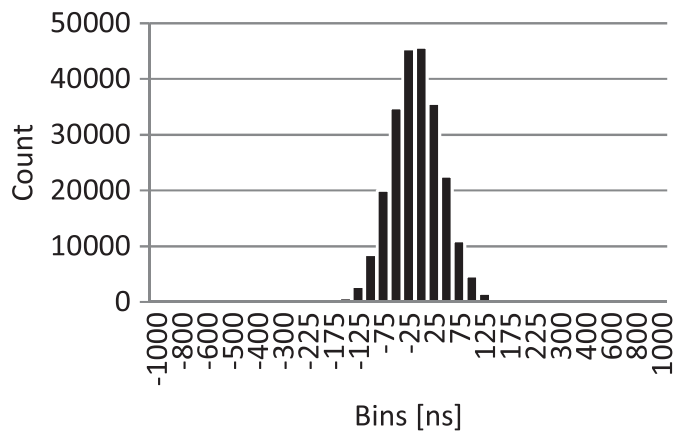

Figure 8. PPS signal delay histogram with PTP IEEE 1588 using COTS Ethernet Switch without traffic.

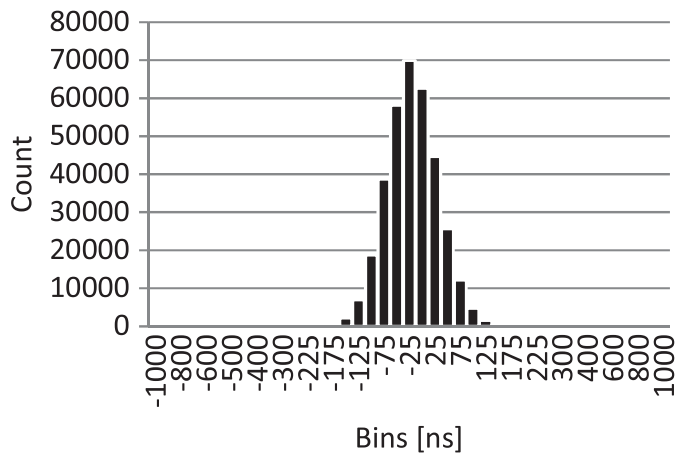

Figure 9. PPS signal delay histogram with PTP IEEE 1588 using COTS Ethernet Switch with 10 Mbit traffic.

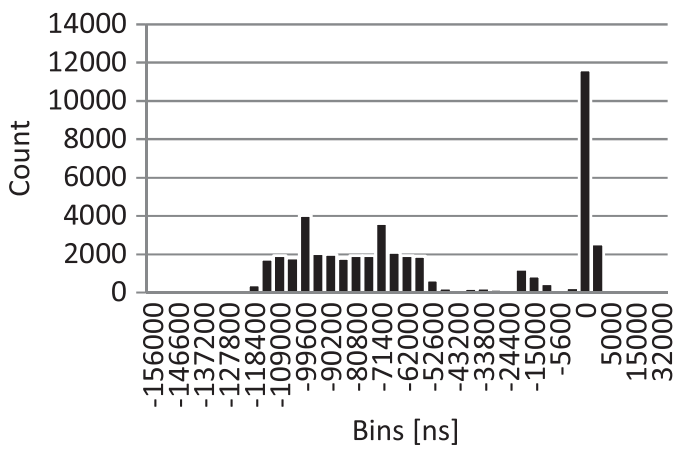

Figure 10. PPS signal delay histogram with PTP IEEE 1588 using COTS Ethernet Switch with 100 Mbit traffic.

\subsection{Delay measurements with PTP}

The time difference between the two PPS signals, F1 and F2, aligned with the second transition of the two clocks that have been synchronized using the Precision Time Protocol IEEE 1588 , was measured with the digital oscilloscope as presented in figure 6.

In figures 7 and 8 , the measurements obtained are reported. The histogram presented in figure 8 represents the time deviations between the two clocks. The measurements shown are gathered over a period of $64 \mathrm{~h}$.

The mean time delay is $44.88 \mathrm{~ns}$ with a standard deviation of $42.03 \mathrm{~ns}$ and a maximum value of $693 \mathrm{~ns}$. Figure 9 shows the histogram of delay measurements between the two PPS signals with 10 Mbit of traffic in the network. 


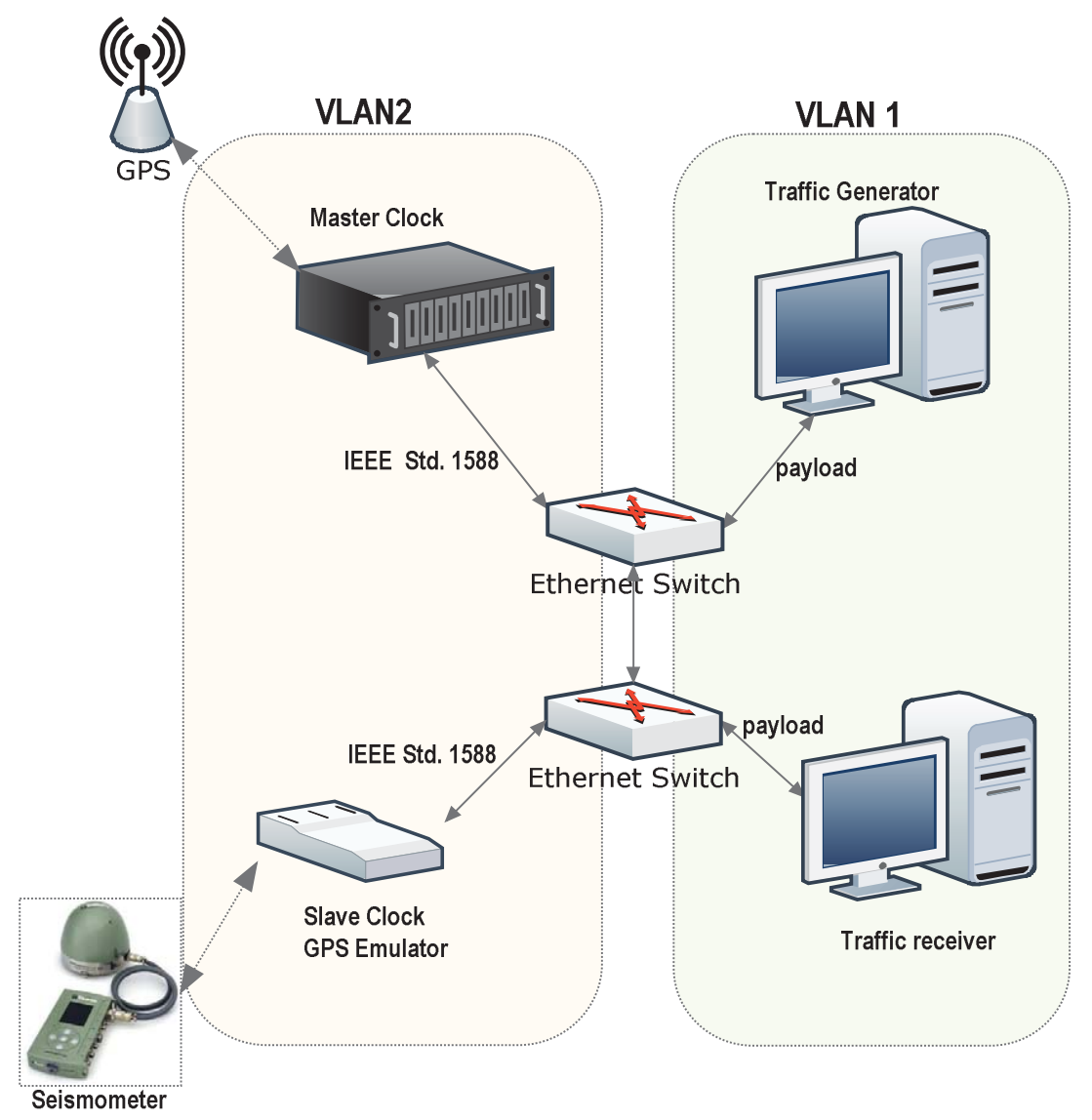

Figure 11. VLAN setup for network components with IEEE 1588.

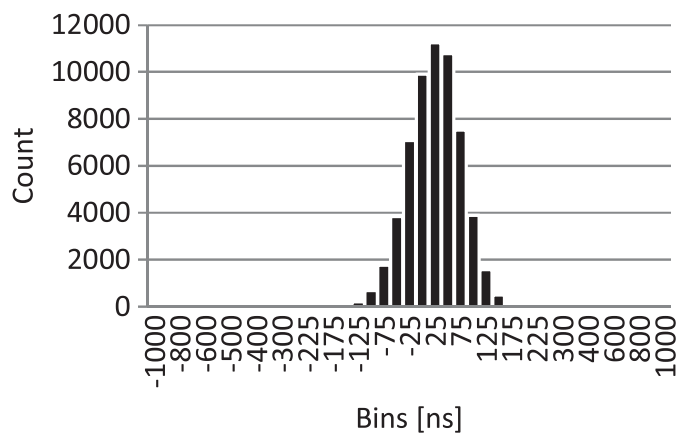

Figure 12. PPS signal delay histogram with PTP IEEE 1588 using COTS Ethernet Switch with 100 Mbit traffic and VLAN setup.

Delays with 10 Mbit traffic have a mean value of approximately $49 \mathrm{~ns}$ with a standard deviation of $45.1 \mathrm{~ns}$ and a maximum delay of 989 ns. Delays are always lower than $1 \mu \mathrm{s}$. traffic

Figure 10 presents the measurements with 100 Mbit

Generating medium and high traffic, the results presented when COTS Ethernet Switch is being used show a time delay mean value higher than the corresponding values measured with the same switch without traffic. Delays with 100 Mbit of network payload take values in the order of hundreds of
Table 2. Experimental results obtained for two PPS signal delay.

\begin{tabular}{lll}
\hline $\begin{array}{l}\text { Network } \\
\text { setup }\end{array}$ & Mean (ns) & $\begin{array}{l}\text { Standard } \\
\text { deviation (ns) }\end{array}$ \\
\hline Without traffic & 44.88 & 42.03 \\
With 10 Mbit of traffic & 49 & 45.1 \\
$\begin{array}{l}\text { With 100 Mbit of } \\
\text { traffic and VLAN setup }\end{array}$ & 43.75 & 42 \\
\hline
\end{tabular}

microseconds as shown in figure 10. The accuracy in the clock adjustment depends on the travel time variability (or jitter) of the synchronization frames. The bigger the jitter, the worse the synchronization. Due to the large payload, the travel time of the PTP frames is variable and some of them were unable to reach their destination as UDP data frames. Both reasons (jitter and lost frames) explain why the PTP does not achieve better accuracy in this setup.

In order to reduce the influence of the high traffic on the PTP frames travel time, the setup presented in figure 11 was created, using Virtual LAN (VLAN) configuration on the switch. In this way, the IEEE 1588 frames between master and slave have been separated virtually from the rest of the network traffic.

The delay measurement histogram with VLAN setup is presented in figure 12. The time delay mean value is $43.75 \mathrm{~ns}$ with a standard deviation of $42 \mathrm{~ns}$ and a maximum 
value of $832 \mathrm{~ns}$. It can be seen that VLAN configuration helps to reduce jitter of the frame's travel time improving the accuracy of the PTP protocol.

Experiments were made to study the stability of the PPS signals and the time synchronization in the Ethernet MSN.

\section{Conclusions}

An experimental setup and the results obtained have been presented in order to evaluate delays between an onshore master clock GPS PPS signal and an underwater synchronization signal generated by a PTP GPS Emulator. The IEEE 1588 protocol was implemented to synchronize the GPS Emulator signal using a GPS synchronized on-shore reference. Delay measurements are made building an Ethernet network with different data traffic payload. Table 2 shows a summary of the measurement as a function of the network setup and of the traffic generated in the network.

With these results one can conclude that IEEE 1588 standard is a valid strategy to synchronize distributed nodes in a Marine Sensor Network where Ethernet is used and instruments with highly accurate clocks such as geophones or hydrophones are required. The results also show that the OBSEA network requires a VLAN setup of switches to separate the IEEE 1588 instrument traffic from PTP synchronization frames.

Underwater seismometers implementing the IEEE 1588 Precision Time Protocol have been developed and validated to work in network systems such as the OBSEA network. We can conclude, after these laboratory tests, that instruments connected to these networks can be synchronized with GPS time through the IEEE 1588 protocol with a precision up to hundreds of nanoseconds.

\section{Acknowledgment}

This work was supported by the Spanish Government under research projects: Interoperability in Environmental and Marine Sensor Networks (CTM2009-08867), Sismòmetre marí digital amb connexió per cable VALTEC09-10059, Centre D’innovació i Desenvolupament Empresarial (CIDEM), Generalitat de Catalunya and 'Integración de sensores para monitorización submarina en una red con sincronización temporal' PT2009-0080.

\section{References}

[1] Bermudez L, Delory E, O'Reilly T, del Rio and Fernandez J 2009 Ocean observing systems demystified Proc. OCEANS 2009, MTS/IEEE Biloxi-Marine Technology for Our Future: Global and Local Challenges (26-29 October 2009) pp 1-7

[2] Ocean Observatory at MBARI (Monterey Bay Aquarium Research Institute) http://www.mbari.org/rd

[3] Ocean Observatories Initiative http://www.oceanleadership. org/programs-and-partnerships/ocean-observing/

[4] Hofmann M 2010 Designing a network for ocean observatories Proc. OCEANS (20-23 September 2010) pp 1-4

[5] Milevsky A and Walrod J 2008 Development and test of IEEE 1588 precision timing protocol for ocean observatory networks Proc. OCEANS (15-18 September 2008) pp 1-7

[6] Lentz S and Lécroart A 2009 Precision timing in the NEPTUNE Canada network Proc. OCEANS 2009 (Europe) pp 1-5

[7] Mánuel-Lázaro A, Nogueras M and del Río J 2010 OBSEA: an expandable seafloor observatory Sea Technol. Mag. 51 37-9

[8] IEEE STD 15882008 Standard for a Precision Clock Synchronization Protocol for Networked Measurement and Control Systems (New York: IEEE)

[9] Paces P, Sipos M and Vesely M 2009 Verification of IEEE1588 time synchronization in NASA agate data bus standard Proc. ICEMI 2009 pp 1-5

[10] Cooklev T, Eidson J C, Fellow L and Pakdaman A 2007 An implementation of IEEE 1588 over IEEE $802.1 \mathrm{lb}$ for synchronization of wireless local area network nodes IEEE Trans. Instrum. Meas. 56 1632-9

[11] Rodríguez I, Mánuel A, Carlosena A, Bermúdez A, del Río J and Shariat-Panahi S 2006 A signal processing in ocean bottom seismographs for refraction seismology IEEE Trans. Instrum. Meas. 55 652-8

[12] André M, van der Schaar M, Zaugg S, Houégnigan L, Sánchez A, Mas A, Morell M, Solé M and Castell J V 2010 Listen to the deep: real-time monitoring of acoustic events Proc. Eur. Conf. Underw. Acoust. vol 1 pp 77-80

[13] Correll K, Barendt N and Branicky M 2005 Design considerations for software only implementations of the IEEE 1588 precision time protocol Conf. on IEEE 1588

[14] InES Institute of Embedded Systems http://www.ines.zhaw.ch/ en/engineering/ines/ieee-1588/overview.html

[15] del Rio J, Toma D, Mànuel A and Ramos H 2009 Evaluation of IEEE 1588 applied to synchronized acquisition in marine sensor networks (MSN) Imeko 19th World Congress (Lisbon, Portugal, September 2009) pp 514-7 